\title{
A correlation analysis between tumor imaging changes and p-AKT and HSP70 expression in tumor cells after osteosarcoma chemotherapy
}

\author{
FENG JI ${ }^{1}$, RAN $\mathrm{LV}^{2}$ and TING ZHAO ${ }^{1}$ \\ ${ }^{1}$ Department of Radiology, Liaocheng People's Hospital; ${ }^{2}$ Department of Medical Oncology, \\ Liaocheng Tumor Hospital, Liaocheng, Shandong 252000, P.R. China
}

Received June 1, 2017; Accepted September 11, 2017

DOI: $10.3892 / \mathrm{ol} .2017 .7005$

\begin{abstract}
This study sought to investigate osteosarcoma property changes after neoadjuvant chemotherapy and to analyze any correlation between changes with phosphoAKT (p-AKT) and heat shock protein 70 (HSP70) expression in osteosarcoma cells. Thirty patients with osteosarcoma treated at Liaocheng People's Hospital between January and October, 2016 were given an imaging examination before and after neoadjuvant chemotherapy to examine osteosarcoma tumor properties, with images scored. Immunohistochemistry was used to determine p-AKT and HSP70 expression levels, as well as tumor cell necrosis rate (TCNR), in specimens obtained before and after chemotherapy. The correlation between the imaging changes of osteosarcoma after chemotherapy and the expressions of p-AKT and HSP70 in tumor cells. Compared with pre-chemotherapy, the imaging scores of the 30 patients significantly increased after chemotherapy $(\mathrm{P}<0.05)$. The radiographic score of the TCNR $\geq 90 \%$ group was $11.3 \pm 0.5$, which was significantly higher than that of the TCNR $<90 \%$ group $(8.7 \pm 0.3, \mathrm{P}<0.05)$. p-AKT expression in osteosarcoma cells was found in $13.3 \%$ of samples (4/30 cases) after chemotherapy, which was significantly lower than prior to chemotherapy $(73.3 \%, 22 / 30$ cases, $\mathrm{P}<0.05)$. After chemotherapy, HSP70 expression in osteosarcoma cells was found in $6.7 \%$ of samples (2/30 cases), which was significantly lower than prior to chemotherapy $(83.3 \%, 25 / 30$ cases, $\mathrm{P}<0.05)$. $\mathrm{p}-\mathrm{AKT}$ and HSP70 expression levels were found to be correlated with TCNR after chemotherapy $(\mathrm{P}<0.05)$. After chemotherapy, p-AKT and HSP70 expression levels demonstrated a positive correlation with TCNR. Tumor property changes, as uncovered by imaging, were significantly inversely correlated with tumor cell p-AKT and HSP70 expression after
\end{abstract}

Correspondence to: Dr Ting Zhao, Department of Radiology, Liaocheng People's Hospital, 67 Dongchangxi Road, Liaocheng, Shandong 252000, P.R. China

E-mail: drtingzhao@163.com

Key words: osteosarcoma, imaging changes, tumor cells, phospho-AKT, heat shock protein 70 chemotherapy. Therefore, osteosarcoma properties, as determined through X-ray imaging, were closely related to $\mathrm{p}$-AKT and HSP70 expression in osteosarcoma cells after neoadjuvant chemotherapy. The effect of chemotherapy can be evaluated by observing the above examination results.

\section{Introduction}

Osteosarcoma grows quickly and is more likely to develop pulmonary metastasis during the early phase. Moreover, patients are prone to multidrug resistance, so there is a certain degree of difficulty present regarding clinical treatment (1). Although chemotherapy and surgery techniques have made great progress in recent years, the 5-year survival rate of osteosarcoma patients has not significantly improved $(2,3)$. For this reason, it is necessary to actively explore new potentially effective treatments for osteosarcoma. Neoadjuvant chemotherapy is a new strategy for tumor treatment developed in recent years. Phospho-AKT (p-AKT) and heat shock protein 70 (HSP70) are important proteins which actively participate in the pathogenesis and development of various diseases such as liver and gastric cancers. At present, there is no data on the relationship between p-AKT and HSP70 protein expression levels in osteosarcoma cells and physical tumor properties after neoadjuvant chemotherapy. The aim of this study was to investigate visible changes to osteosarcomas after neoadjuvant chemotherapy and to determine whether any correlation between these changes and osteosarcoma cell p-AKT and HSP70 expression exists, in order to provide a theoretical basis for the clinical treatment of osteosarcoma.

\section{Materials and methods}

General patient information. Thirty patients with osteosarcoma admitted to Liaocheng People's Hospital between January and October, 2016 were selected as subjects. Inclusion criteria in the study were as follows: i) patients with osteosarcoma confirmed by pathological examination (4); ii) patients who received the same new auxiliary chemotherapy regimen before operation; iii) patients and/or guardians who signed informed consent. Exclusion criteria were as follows: i) patients who presented osteosarcoma in combination with other cancers; ii) patients suffering from heart, brain, and other vital organ 
Table I. Criteria for image scoring of osteosarcoma patients.

\begin{tabular}{llll}
\hline Imaging characteristic & \multicolumn{1}{c}{0 points } & 1 point & 2 points \\
\hline Bone calcification & None & Partial & Complete \\
Tumor boundaries & Not clear & Clear & Obviously clear \\
Bone shell formation & None & Partially formed & Completely repaired \\
Osteolytic distruction area & Obvious & Partially present & None or completely repaired \\
Pathological fractures & Clearly present & Callus formation in broken ending & Healing or no pathological fracture \\
Periosteal reaction & Obvious & Less & None \\
Soft tissue mass & Obvious & Less & None \\
\hline
\end{tabular}

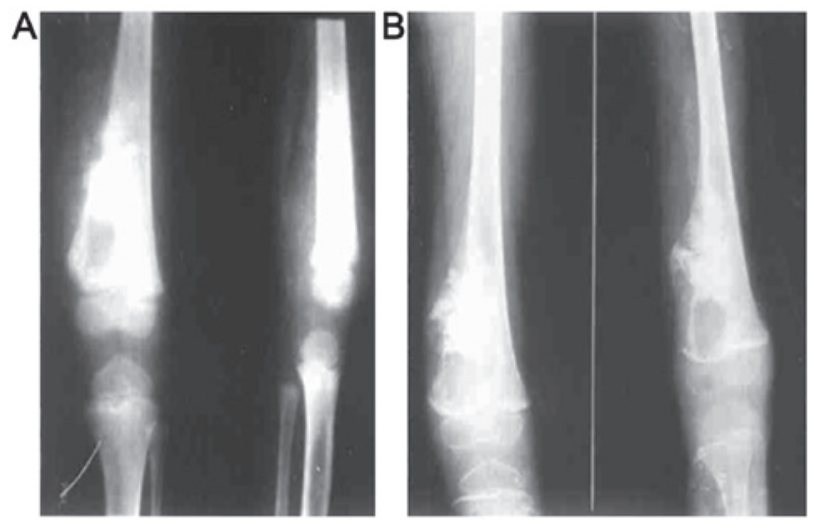

Figure 1. Cortical bone damage. Distal femoral osteosarcoma imaging before and after chemotherapy (A) Mixed bone destruction with periosteal reaction, soft tissue swelling, and blurred boundaries before chemotherapy. (B) Significant calcification and ossification in distal femoral osteosarcoma lesions with clear boundaries and free of soft tissue swelling after chemotherapy.

diseases; iii) patients who were non-compliant with treatment. The thirty subjects selected (18 males and 12 females, aged 18-37 years, mean age $23.4 \pm 3.5$ years) presented 14 cases of osteosarcoma in the distal femur, 7 cases in the upper tibia, 5 cases in the proximal femur, 2 cases in the iliac crest, and 2 cases in the proximal humerus. Treatment regimen was as follows: patients received two courses of neoadjuvant chemotherapy MMIA (6 weeks) before operation, rested for 14 days prior to surgery, and then resumed chemotherapy after operation. The study was approved by the Ethics Committee of Liaocheng People's Hospital.

Imaging examinations before and after chemotherapy. All patients received conventional X-ray examinations before chemotherapy and after 2 courses of chemotherapy.

Osteosarcoma imaging score. The osteosarcoma imaging was scored as outlined in Table I, and the imaging features of the patients before and after chemotherapy were quantified according to the criteria presented in Table I.

\section{Pathological examinations and determination of $p$-AKT and} HSP70 expression

Hematoxylin and eosin $(H \& E)$ and immunohistochemical staining. Needle biopsies were obtained from all patients before chemotherapy, embedded in wax blocks, and collected. Tumor specimens were obtained from all patients during the course of the operation after chemotherapy were also embedded in wax blocks. All blocks were sectioned and subjected to H\&E, p-AKT and HSP70 immunohistochemistry staining. p-AKT, HSP70 and other relevant immunohistochemical kits were purchased from Shanghai Fusheng Industrial Co., Ltd. (Shanghai, China). Sections known to present positive-expression positive controls and phosphate-buffered saline (PBS) negative controls were generated simultaneously.

Immunohistochemical staining evaluation criteria. Ten casual fields of view were observed under microscopy, with yellow or brown particles in the nuclei or cytoplasm with clear backgrounds being considered as positive staining. In addition, samples showing positive staining were divided into strong staining ( $>95 \%$ positive cells), moderate staining (51-95\% positive cells), weak staining (2-50\% positive cells), and absence of expression ( $<2 \%$ positive cells) categories (5). In this study, weak staining and absence of expression were interpreted as negative expression, and strong staining and moderate staining as positive expression.

Evaluation of tumor cell necrosis rate (TCNR). TCNR was calculated in accordance with previously published literature (6) for all patients with preoperative chemotherapy.

Statistical analysis. Statistical analysis was performed using SPSS 20.0 (IBM, Armonk, NY, USA). The data were expressed as mean $\pm \mathrm{SD}$. The enumeration data were analyzed by paired samples t-tests, and measurement data were compared using $\chi^{2}$ tests. The correlation analyses between p-AKT and HSP70 expression levels, TCNR, and image scoring were performed with $\chi^{2}$ tests. A $\mathrm{P}<0.05$ was considered as statistically significant.

\section{Results}

Changes in osteosarcoma characteristics before and after chemotherapy. In this study, osteogenic changes were observed in 20 patients, osteoporosis in 6 cases, and a mixture of the two in 4 cases before chemotherapy. All patients exhibited typical osteosarcoma osteolytic or sclerotic changes, with the tumor border being unclear with cortical bone damage. An example of visible changes to distal femoral osteosarcoma properties before and after chemotherapy is shown in Fig. 1. An example of visible changes to iliac osteosarcoma properties are shown in Fig. 2.

Imaging showed a significant increase in radiographic score after chemotherapy $(\mathrm{P}<0.05)$ compared with before chemotherapy (Table II). 
Table II. Imaging scores for all 30 patients before and after chemotherapy.

Patient number

\begin{tabular}{llllllllllllllllllllllllllllllllllllll} 
Time-points & 1 & 2 & 3 & 4 & 5 & 6 & 7 & 8 & 9 & 10 & 11 & 12 & 13 & 14 & 15 & 16 & 17 & 18 & 19 & 20 & 21 & 22 & 23 & 24 & 25 & 26 & 27 & 28 & 29 & 30 \\
\hline $\begin{array}{l}\text { Before } \\
\text { chemotherapy }\end{array}$ & 4 & 4 & 7 & 5 & 6 & 8 & 7 & 6 & 7 & 6 & 4 & 5 & 5 & 6 & 7 & 8 & 5 & 7 & 8 & 7 & 6 & 6 & 6 & 4 & 4 & 5 & 5 & 7 & 8 & 7 \\
$\begin{array}{l}\text { After } \\
\text { chemotherapy }\end{array}$ & 8 & 7 & 9 & 10 & 11 & 12 & 10 & 9 & 11 & 10 & 8 & 8 & 7 & 12 & 10 & 11 & 8 & 11 & 12 & 10 & 9 & 9 & 10 & 8 & 8 & 9 & 8 & 10 & 12 & 11
\end{tabular}

Table III. Expression of p-AKT and HSP70 in osteosarcoma before and after chemotherapy.

\begin{tabular}{lcccr}
\hline & & \multicolumn{2}{c}{$\mathrm{p}$-AKT } & \multicolumn{2}{c}{ HSP70 } \\
\cline { 3 - 5 } Time-points & $\mathrm{n}$ (cases) & Positive & Negative & Positive \\
\hline Before chemotherapy & 30 & 22 & 8 & 5 \\
After chemotherapy & 30 & 4 & 26 & 25 \\
$\chi^{2}$ & & 8.425 & 9.107 & 28 \\
P-value & & $<0.05$ & $<0.05$ & \\
\hline
\end{tabular}

HSP70, heat shock protein 70 .
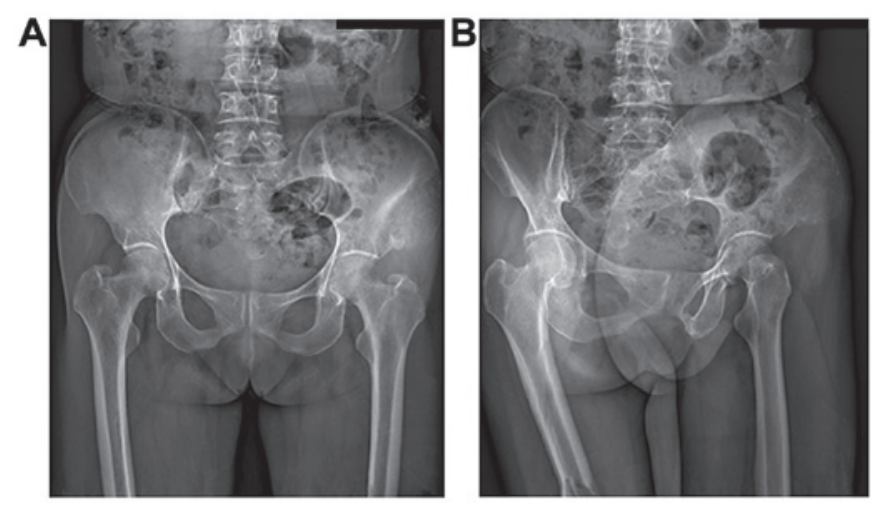

Figure 2. Iliac osteosarcoma imaging before and after chemotherapy (A) Osteolytic destruction with unclear boundaries and local cortical bone fracture before chemotherapy. (B) Clear tumor boundaries, local hardening, and increase of bone fracture healing and continuity were observed in iliac osteosarcoma after chemotherapy.

The relationship between imaging score changes and TCNR after chemotherapy. A TCNR $\geq 90 \%$ indicated good patient prognosis (7). Patients were divided into TCNR $\geq 90 \%(\mathrm{n}=21)$ and TCNR $<90 \%$ groups $(n=9)$, with statistical analysis showing that the average imaging score of the TCNR $\geq 90 \%$ group was $11.3 \pm 0.5$ points, which was significantly higher than that of the TCNR $<90 \%$ group $(8.7 \pm 0.3, \mathrm{P}<0.05)$ (Fig. 3).

The relationship between the expression of $p$-AKT and HSP70 in tumor cells and TCNR after chemotherapy. Positive staining for p-AKT and HSP70 proteins manifested as purple particles in the cytoplasm (Figs. 4 and 5). The positive expression rate of p-AKT in osteosarcoma cells was $13.3 \%$ (4 of 30 cases), which was significantly lower than the $73.3 \%$ found before

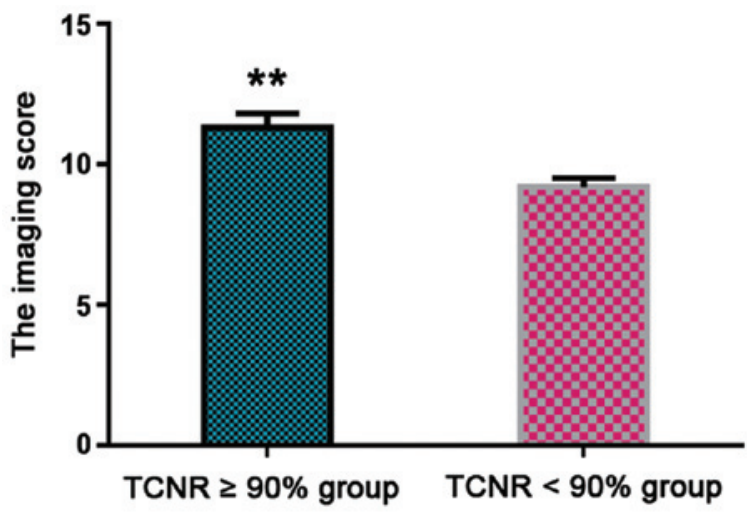

Figure 3. The relationship between imaging score changes and tumor cell necrosis rate (TCNR) after chemotherapy. The imaging score of patients in the TCNR $\geq 90 \%$ group was significantly higher than those in the TCNR $<90 \%$ group $\left({ }^{* *} \mathrm{P}<0.05\right)$.

chemotherapy (22 of 30 cases, $\mathrm{p}<0.05$ ). After chemotherapy, the positive expression rate of HSP70 in osteosarcoma cells was $6.7 \%$ (2 of 30 cases), which was significantly lower than the $83.3 \%$ found before chemotherapy (25 of 30 cases, $\mathrm{P}<0.05$ ) (Table III).

The expression of p-AKT and HSP70 was inversely correlated with TCNR after chemotherapy $(\mathrm{P}<0.05)$. The lower the expression of p-AKT and HSP70, the higher the patient TCNR value (Table IV).

Relationship between imaging score and $p$-AKT and HSP70 expression after chemotherapy. Based on previous comprehensive clinical practice, a postoperative radiographic score $\geq 9$ points was considered as effective chemotherapy, on the contrary, a score of $<9$ points was considered ineffective (8). 

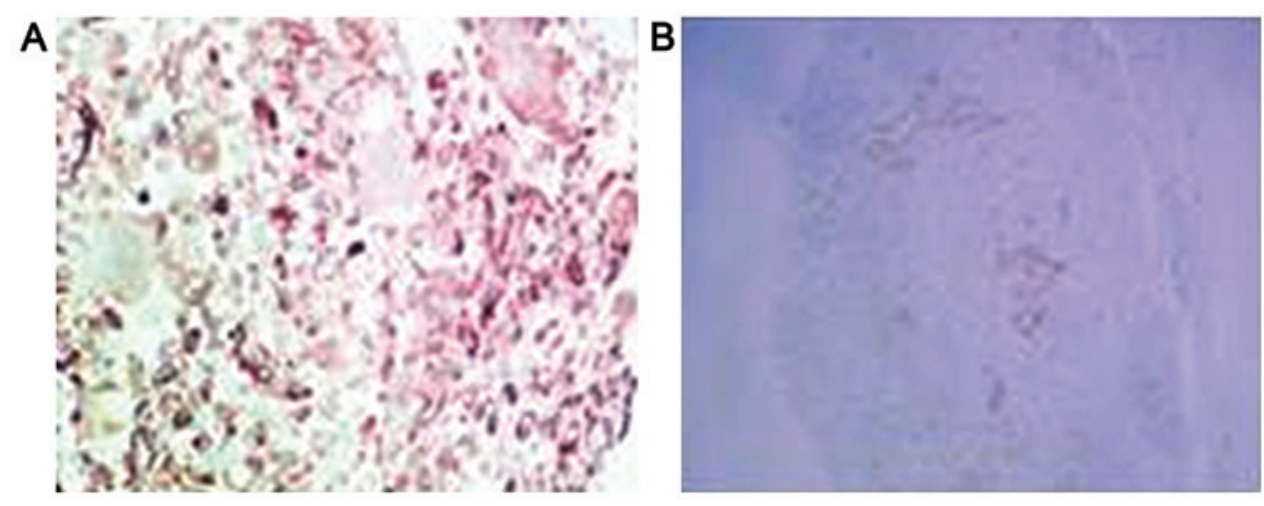

Figure 4. p-AKT expression in osteosarcoma cells detected by immunohistochemistry. (A) Positive expression of p-AKT in osteosarcoma cells. B) Negative expression of p-AKT in osteosarcoma cells.
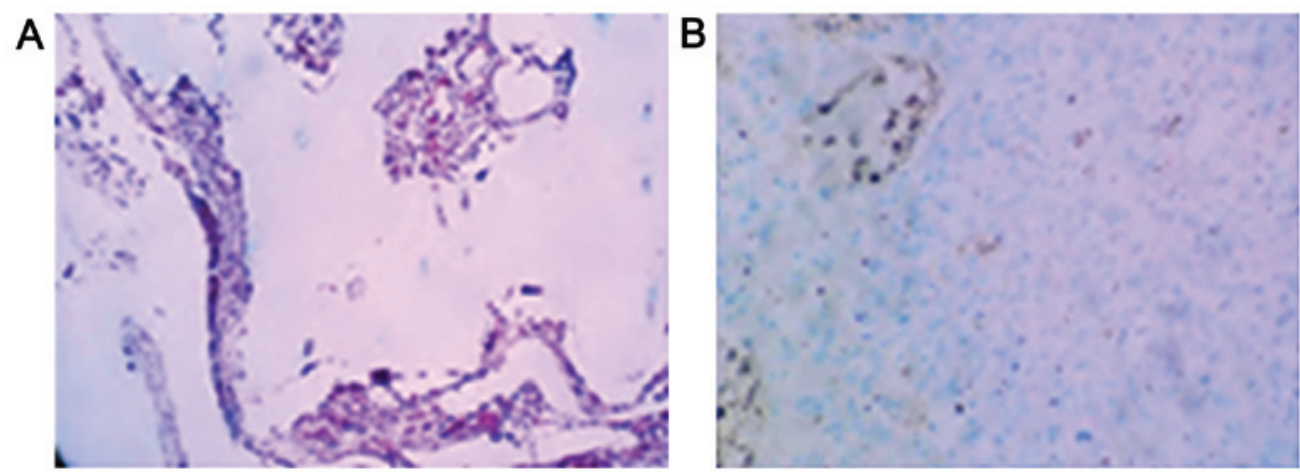

Figure 5. Heat shock protein 70 (HSP70) expression in osteosarcoma cells detected by immunohistochemistry. (A) Positive expression of HSP70 in osteosarcoma cells. (B) Negative expression of HSP70 in osteosarcoma cells.

Table IV. The relationship between expression of p-AKT and HSP70 and TCNR after chemotherapy.

\begin{tabular}{lcccccc}
\hline & \multicolumn{2}{c}{$\mathrm{p}$-AKT expression } & & \multicolumn{2}{c}{ HSP70 expression } \\
\cline { 2 - 3 } \cline { 6 - 7 } TCNR & Positive & Negative & & Positive & Negative \\
\hline $290 \%$ & 2 & 19 & & 1 & 18 \\
$<90 \%$ & 2 & 7 & & 1 & 10 \\
$\chi^{2}$ & 6.128 & & & 7.603 & \\
P-value & $<0.05$ & & & $<0.05$ & \\
\hline
\end{tabular}

TCNR, tumor cell necrosis rate; HSP70, heat shock protein 70.

Table V. Patient imaging score and expression of p-AKT and HSP70 after chemotherapy.

\begin{tabular}{lccccc}
\hline & \multicolumn{2}{c}{ p-AKT expression } & & \multicolumn{2}{c}{ HSP70 expression } \\
\cline { 2 - 3 } \cline { 5 - 6 } Score & Positive & Negative & & Positive & Negative \\
\hline$\geq 9$ points & 2 & 19 & & 1 & 18 \\
$<9$ points & 2 & 7 & & 1 & 10 \\
$\chi^{2}$ & 6.128 & & & 7.603 & \\
P-value & $<0.05$ & & & $<0.05$ & \\
\hline
\end{tabular}

HSP70, heat shock protein 70 .
Imaging score was inversely correlated with the expression of p-AKT and HSP70 in tumor cells after chemotherapy. The higher the imaging score, the lower the expression rates of p-AKT and HSP70 (Table V).

\section{Discussion}

Neoadjuvant chemotherapy improves 5-year survival rate for osteosarcoma $(9,10)$. After patients with osteosarcoma receive chemotherapy, tumor cells undergo necrosis, tumor tissue is directly absorbed by the body, and the gaps left are replaced by new bone (11-13), leading to increased complexity with regards to imaging results after osteosarcoma chemotherapy. The occurrence and development of osteosarcoma is closely related to cellular molecular expression levels. Moreover, changes at the molecular level are related to morphological changes which can be detected by imaging (14-17).

This study showed that typical osteosarcoma osteolytic or sclerotic changes combined with cortical bone damage occurred in all 30 patients after chemotherapy, which was consistent with previous findings (18). These changes may be related to alterations in osteosarcoma pathogenesis. After scoring patient images, we found that the imaging scores of all 30 patients significantly increased after chemotherapy $(\mathrm{P}<0.05)$. The radiographic score of the TCNR $\geq 90 \%$ group was significantly higher than that of the TCNR $<90 \%$ group $(\mathrm{P}<0.05)$, suggesting that imaging score was positively correlated with TCNR, and accordingly, with improved patient 
prognosis. Therefore, imaging-detected characteristic changes can be used to predict patient chemotherapy effectiveness, thus providing a theoretical basis for clinical treatment (19).

AKT belongs to a class of important proteins in the PI3K/AKT signaling pathway, and AKT activation is dependent on the activation of upstream PI3K. p-AKT is the activated form of AKT (20). Previous data showed (21) that abnormal expression of $\mathrm{p}-\mathrm{AKT}$ was found in laryngeal squamous cell carcinoma, gastric cancer, and other cancers. Heat shock proteins are stress proteins which regulate the activity and physiological function of a wide variety of proteins. HSP70 is an important member of the heat shock protein family (22). A previous study suggested (23) that HSP70 was abnormally expressed in cervical cancer, nasopharyngeal carcinoma, colorectal, and other cancers, and involved in the occurrence and development of cancers.

This study showed that the positive expression rate of p-AKT in osteosarcoma cells was $13.3 \%$ (4 of 30 cases) after chemotherapy, which was significantly lower than the $73.3 \%$ found before chemotherapy ( 22 of 30 cases, $\mathrm{P}<0.05$ ). The positive expression rate of HSP70 in osteosarcoma cells was $6.7 \%$ ( 2 of 30 cases) after chemotherapy, which was significantly lower than the $83.3 \%$ found before chemotherapy ( 25 of 30 cases, $\mathrm{P}<0.05$ ), suggesting that neoadjuvant chemotherapy drugs could effectively inhibit the proliferation of osteosarcoma cells. We also found that the expressions of p-AKT and HSP70 were correlated with TCNR after chemotherapy $(\mathrm{P}<0.05)$. The lower the expression rates of $\mathrm{p}-\mathrm{AKT}$ and HSP70 were, the higher the TCNR was, suggesting that the expression of p-AKT and HSP70 in osteosarcoma cells after neoadjuvant chemotherapy could be used to predict the efficacy of neoadjuvant chemotherapy. In addition, imaging scores were significantly correlated with the expression rates of p-AKT and HSP70 in tumor cells. The higher the imaging score was, the lower the expression rates of p-AKT and HSP70 were, which implied that image analysis after neoadjuvant chemotherapy could be used to posit the expressions of $\mathrm{p}-\mathrm{AKT}$ and HSP70 in patient tumor cells, and thus could be used to predict and evaluate the efficacy of neoadjuvant chemotherapy.

In conclusion, osteosarcoma characteristics, as detected by imaging, after neoadjuvant chemotherapy were closely related to the expressions of p-AKT and HSP70 in osteosarcoma cells. The effect of chemotherapy can be evaluated by observing the above examination results.

\section{References}

1. Laux CJ, Berzaczy G, Weber M, Lang S, Dominkus M, Windhager R, Nöbauer-Huhmann IM and Funovics PT: Tumour response of osteosarcoma to neoadjuvant chemotherapy evaluated by magnetic resonance imaging as prognostic factor for outcome. Int Orthop 39: 97-104, 2015.

2. Kubo T, Furuta T, Johan MP, Adachi N and Ochi M: Percent slope analysis of dynamic magnetic resonance imaging for assessment of chemotherapy response of osteosarcoma or Ewing sarcoma: Systematic review and meta-analysis. Skeletal Radiol 45: 1235-1242, 2016.

3. Li H, Li XY, Bu J and Xiao T: How to explain the role of magnetic resonance imaging on evaluating tumour response of osteosarcoma to neoadjuvant chemotherapy? Int Orthop 39: 1031-1032, 2015

4. Ji WP and He NB: Investigation on the DNA repaired gene polymorphisms and response to chemotherapy and overall survival of osteosarcoma. Int J Clin Exp Pathol 8: 894-899, 2015.
5. Hagleitner MM, Coenen MJ, Gelderblom H, Makkinje RR, Vos HI, de Bont ES, van der Graaf WT, Schreuder HW, Flucke U, van Leeuwen FN, et al: A first step towards personalized medicine in osteosarcoma: Pharmacogenetics as predictive marker of outcome after chemotherapy based treatment. Clin Cancer Res 21: 3436-3441, 2015.

6. Sarman H, Bayram R and Benek SB: Anticancer drugs with chemotherapeutic interactions with thymoquinone in osteosarcoma cells. Eur Rev Med Pharmacol Sci 20: 1263-1270, 2016.

7. Li S, Sun W, Wang H, Zuo D, Hua Y and Cai Z: Research progress on the multidrug resistance mechanisms of osteosarcoma chemotherapy and reversal. Tumour Biol 36: 1329-1338, 2015.

8. Wang X, Zheng H, Shou T, Tang C, Miao K and Wang P: Effectiveness of multi-drug regimen chemotherapy treatment in osteosarcoma patients: A network meta-analysis of randomized controlled trials. J Orthop Surg Res 12: 52, 2017.

9. Zhu XZ and Mei J: Effect and mechanism analysis of siRNA in inhibiting VEGF and its anti-angiogenesis effects in human osteosarcoma bearing rats. Eur Rev Med Pharmacol Sci 19: 4362-4370, 2015

10. Ahn JH, Cho WH, Lee JA, Kim DH, Seo JH and Lim JS: Bone mineral density change during adjuvant chemotherapy in pediatric osteosarcoma. Ann Pediatr Endocrinol Metab 20: 150-154, 2015.

11. Byun BH, Kim SH, Lim SM, Lim I, Kong CB, Song WS, Cho WH, Jeon DG, Lee SY, Koh JS, et al: Prediction of response to neoadjuvant chemotherapy in osteosarcoma using dual-phase (18)F-FDG PET/CT. Eur Radiol 25: 2015-2024, 2015.

12. 1Chang KJ, Kong CB, Cho WH, Jeon DG, Lee SY, Lim I and Lim SM: Usefulness of increased 18F-FDG uptake for detecting local recurrence in patients with extremity osteosarcoma treated with surgical resection and endoprosthetic replacement. Skeletal Radiol 44: 529-537, 2015.

13. Avril P, Le Nail LR, Brennan MÁ, Rosset P, De Pinieux G, Layrolle P, Heymann D, Perrot P and Trichet V: Mesenchymal stem cells increase proliferation but do not change quiescent state of osteosarcoma cells: Potential implications according to the tumor resection status. J Bone Oncol 5: 5-14, 2015.

14. Cai S, Zhang T, Zhang D, Qiu G and Liu Y: Volume-sensitive chloride channels are involved in cisplatin treatment of osteosarcoma. Mol Med Rep 11: 2465-2470, 2015.

15. Guo J, Glass JO, McCarville MB, Shulkin BL, Daryani VM, Stewart CF, Wu J, Mao S, Dwek JR, Fayad LM, et al: Assessing vascular effects of adding bevacizumab to neoadjuvant chemotherapy in osteosarcoma using DCE-MRI. Br J Cancer 113: 1282-1288, 2015

16. Xiao X, Wang W, Zhang H, Gao P, Fan B, Huang C, Fu J, Chen G, Shi L, Zhu H, et al: Individualized chemotherapy for osteosarcoma and identification of gene mutations in osteosarcoma. Tumour Biol 36: 2427-2435, 2015.

17. Lee JA, Jeon DG, Cho WH, Song WS, Yoon HS, Park HJ, Park BK, Choi HS, Ahn HS, Lee JW, et al: Higher gemcitabine dose was associated with better outcome of osteosarcoma patients receiving gemcitabine-docetaxel chemotherapy. Pediatr Blood Cancer 63: 1552-1556, 2016.

18. Katagiri H, Sugiyama H, Takahashi M, Murata H, Wasa J, Hosaka S and Miyagi M: Osteosarcoma of the pelvis treated successfully with repetitive intra-arterial chemotherapy and radiation therapy: A report of a case with a 21-year follow-up. J Orthop Sci 20: 568-573, 2015.

19. Kleinerman E: Maximum benefit of chemotherapy for osteosarcoma achieved-what are the next steps? Lancet Oncol 17: 1340-1342, 2016

20. Fang $X$, Jiang Y, Feng L, Chen H, Zhen C, Ding M and Wang X: Blockade of PI3K/AKT pathway enhances sensitivity of Raji cells to chemotherapy through down-regulation of HSP70. Cancer Cell Int 13: 48, 2013.

21. Chatterjee M, Andrulis M, Stühmer T, Müller E, Hofmann C, Steinbrunn T, Heimberger T, Schraud H, Kressmann S, Einsele H, et al: The PI3K/Akt signaling pathway regulates the expression of Hsp70, which critically contributes to Hsp90-chaperone function and tumor cell survival in multiple myeloma. Haematologica 98: 1132-1141, 2013.

22. Boon E, van der Graaf WT, Gelderblom H, Tesselaar ME, van Es RJ, Oosting SF, de Bree R, van Meerten E, Hoeben A, Smeele LE, et al: Impact of chemotherapy on the outcome of osteosarcoma of the head and neck in adults. Head Neck 39: 140-146, 2017.

23. Pan T, Li X, Xie W, Jankovic J and Le W: Valproic acid-mediated Hsp70 induction and anti-apoptotic neuroprotection in SH-SY5Y cells. FEBS Lett 579: 6716-6720, 2005. 